\title{
Effectiveness of seasonal 2012/13 vaccine in preventing laboratory-confirmed influenza infection in primary care in the United Kingdom: mid-season analysis 2012/13
}

J McMenamin (jim.mcmenamin@nhs.net) ${ }^{1}$, N Andrews ${ }^{2}$, C Robertson $^{1,3,4}$, D M Fleming ${ }^{5}$, H Durnall ${ }^{5}$, B von Wissmann ${ }^{1}$, J Ellis ${ }^{6}$,

A Lackenby ${ }^{6}$, S Cottrell'7, B Smyth ${ }^{8}$, M Zambon $^{2}$, C Moore $^{7}$, J M Watson ${ }^{2}$, R G Pebody $^{2}$

1. Health Protection Scotland, Glasgow, United Kingdom

2. Health Protection Agency Health Protection Services - Colindale, London, United Kingdom

3. University of Strathclyde, Glasgow, United Kingdom

4. International Prevention Research Institute, Lyon, France

5. Royal College of General Practitioners Research and Surveillance Centre, Birmingham, United Kingdom

6. Health Protection Agency Microbiology Services - Colindale, London, United Kingdom

7. Public Health Wales, Cardiff, United Kingdom

8. Public Health Agency Northern Ireland, Belfast, United Kingdom

Citation style for this article:

McMenamin J, Andrews N, Robertson C, Fleming DM, Durnall H, von Wissmann B, Ellis J, Lackenby A, Cottrell S, Smyth B, Zambon M, Moore C, Watson JM, Pebody RG. Effectiveness of seasonal $2012 / 13$ vaccine in preventing laboratory-confirmed influenza infection in primary care in the United Kingdom: mid-season analysis 2012/13. Euro Surveill. 2013;18(5):pii=20393. Available online: http://www.eurosurveillance.org/ViewArticle.aspx?Articleld=20393

Article published on 31 January 2013

The early experience of the United Kingdom (UK) is that influenza $B$ has dominated the influenza 2012/13 season. Overall trivalent influenza vaccine (TIV) adjusted vaccine effectiveness (VE) against all laboratory-confirmed influenza in primary care was $51 \%$ ( $95 \%$ confidence interval (CI): $27 \%$ to $68 \%$ ); TIV adjusted VE against influenza A alone or influenza B alone was $49 \%(95 \% \mathrm{Cl}:-2 \%$ to $75 \%)$ and $52 \%(95 \%$ $\mathrm{Cl}: 23 \%$ to $70 \%$ ) respectively. Vaccination remains the best protection against influenza.

\section{Background}

In common with many countries the United Kingdom (UK) experienced unusually late influenza activity in 2011/12, with activity peaking only in week 8 of 2012 [1] in a season dominated by influenza $A\left(\mathrm{H}_{3} \mathrm{~N}_{2}\right)$ and characterised by excess in all-cause mortality and the occurrence of influenza outbreaks in nursing home settings $[2,3]$. Trivalent seasonal influenza vaccine (TIV) provided only moderate initial protection against $\mathrm{A}\left(\mathrm{H}_{3} \mathrm{~N}_{2}\right)$ infection in primary care in $2011 / 12$ with subsequent significant intra-seasonal waning of protection [1]. Overall in 2011/12 TIV VE against confirmed $\mathrm{A}\left(\mathrm{H}_{3} \mathrm{~N}_{2}\right)$ infection, adjusted for age, surveillance scheme and month was $23 \%$ (95\% confidence interval (CI): $-10 \%$ to $47 \%)$.

These results, coupled with virological data on emerging strain types, supported the rationale for the change to the World Health Organization (WHO) recommendation for composition of the TIV for the northern hemisphere season for 2012/13 [4] in which the $A / C a l i f o r n i a / 7 / 2009 \quad\left(\mathrm{H}_{1} \mathrm{~N}_{1}\right)$ pdmo9-like virus was retained, and the $\mathrm{A}\left(\mathrm{H}_{3} \mathrm{~N}_{2}\right)$ vaccine strain was updated to an $A /$ Victoria/361/2011 ( $\left.\mathrm{H}_{3} \mathrm{~N}_{2}\right)$-like virus. Additionally, the $B /$ Victoria lineage influenza $B$ vaccine component was replaced with an influenza B strain of the $B /$ Yamagata lineage (B/Wisconsin/1/2010-like virus). Compared to season $2011 / 12$, the UK has experienced a relatively early influenza season in 2012/13, which has been dominated by influenza $B$ circulation, but also with some $\mathrm{A}\left(\mathrm{H}_{3} \mathrm{~N}_{2}\right)$ circulation [5]. The 2012/13 intra-seasonal estimation of vaccine uptake in individuals who are in clinical groups at increased clinical risk of complications [6] has indicated similar levels compared to the same time point in 2011/12 [5]. The UK has an established surveillance scheme to produce interim and end of season estimation of the effectiveness of the influenza vaccine programme $[1,7]$ and this paper presents the interim evaluation of the effectiveness of the $2012 / 13$ vaccine.

\section{Estimation of influenza vaccine effectiveness}

Study population and period

Data were derived from five primary care influenza sentinel surveillance schemes in England (two schemes), Northern Ireland, Scotland and Wales. Details of the Royal College of General Practitioners (RCGP), Health Protection Agency (HPA) Specialist Microbiology Network (SMN), Public Health Wales, Public Health Agency of Northern Ireland and Health Protection Scotland (HPS) swabbing schemes have been presented previously $[1,7]$.

The study period ran from 1 October 2012 to 4 January 2013. Cases were defined, as previously $[1,7]$, as persons presenting during the study period in a participating general practitioner (GP) practice with an acute influenza-like illness (ILI) who were swabbed and then tested positive for influenza A or influenza B. ILI was 
defined as an individual presenting in primary care with an acute respiratory illness with either history of fever or documented temperature $>38^{\circ} \mathrm{C}$ or complaint of feverishness $[1,7]$. Patients were swabbed as part of clinical care, with verbal consent. Controls were individuals presenting with ILI in the same period that were swabbed and tested negative for influenza.

A standardised questionnaire collecting demographic, clinical and epidemiological information from cases and controls including date of birth, sex, defined underlying clinical risk group [1,7], date of onset of respiratory illness, date of specimen and influenza vaccination status for $2012 / 13$ with vaccination dates was completed by the patient's responsible GP at the time of swabbing.

\section{Laboratory methods}

Laboratory confirmation was undertaken using realtime polymerase chain reaction (RT-PCR) assays for circulating influenza A viruses, influenza B viruses and other respiratory viruses [8,9]. Samples in England were sent to the HPA Microbiology Services, Colindale (RCGP scheme) or one of the specialist HPA microbiology laboratories (SMN scheme). Samples in Wales were sent to the Public Health Wales Specialist Virology Centre and in Scotland to the West of Scotland Specialist Virology Centre (HPS scheme) for molecular testing. In Northern Ireland samples were sent to the Regional Virus Laboratory, Belfast. All participating UK laboratories are a designated WHO National Influenza Centre (NIC) and participate in WHO and UK quality assurance programmes. This aids RT-PCR assays to be comparable between laboratories.

\section{Statistical methods}

Persons with a date of onset between 1 October 2012 and 4 January 2013 were available for this analysis. If date of onset of symptoms was missing then the date the swab was taken was used to define the time of ILI. Persons were defined as vaccinated if the date of vaccination with the $2012 / 13$ TIV was 14 or more days before onset of illness. Those in whom the period between vaccination and onset of illness was less than 14 days were excluded, as immunity is unknown. Patients were also excluded if the date of vaccination was missing, and samples with a delay greater than 29 days between onset of illness (where known) and sample collection were excluded as the sensitivity of the polymerase chain reaction (PCR) test reduces for long intervals between onset and sampling $[10,11]$.

VE was estimated as 1-(odds ratio) using multivariable logistic regression models with influenza $A$ or influenza B PCR results as outcomes and seasonal vaccination status as the linear predictor. In the analyses evaluating VE in preventing influenza $A$ infection, samples positive for influenza B were excluded and vice versa. In the multivariable analysis the known confounders age (coded into five standard age groups, $>5$ years, 5-14 years, 15-44 years, 45-64 years and $\geq 65$ years) and month of ILI onset (or swab taken if onset was unknown) were included as well as sex and surveillance scheme (HPS, RCGP, SMN, Northern Ireland, Wales). Effect modification by age and scheme was assessed by likelihood ratio tests.

All statistical analyses were carried out in Stata version 12 (StataCorp, College Station, Texas).

\section{2/13 influenza vaccine effectiveness}

This report has information on 1,865 individuals from whom samples were collected during the study period. Of these, 957 samples were collected through the RCGP surveillance scheme, 293 through the SMN scheme, 511 through the HPS scheme, 41 through the Public Health Wales scheme and 63 in Northern Ireland. Table 1 shows the distribution and completeness of the baseline characteristics of the study participants according to whether they were cases or controls.

Those excluded from the study because of late swabbing, a time of less than 14 days between vaccination and onset of symptoms and missing information on vaccination are summarised in Table 2 . There were therefore 1,324 persons (i.e. 121 influenza $A$ cases and 1,203 controls) for whom data on both vaccination status and influenza A infection were available. Similarly, there were 1,580 persons (i.e. 377 influenza B cases and 1,203 controls) included in the estimation of trivalent vaccine for prevention of influenza $B$.

Vaccine effectiveness in prevention of influenza Table 3 shows the number of samples positive and negative for influenza $A$, influenza $B$ and the combined influenza A or B virus according to vaccination status. Crude and adjusted vaccine effectiveness are also shown.

The adjusted VE estimates (Table 3) were 49\% (95\% $\mathrm{Cl}:-2 \%$ to $75 \%)$ for influenza A, $52 \%$ (95\% Cl: $23 \%$ to $70 \%$ ) for influenza $\mathrm{B}$ and $51 \%$ (95\% Cl: $27 \%$ to 68 ) for influenza $A$ and $B$ combined. As seen in previous years, age and month of onset were associated with positivity and vaccination status and were therefore confounding variables. Risk group was missing for $158 / 1,865$ (8.5\%) and this variable was not included in the model as it was not significantly associated with swab positivity when added to the multivariable model and analyses in previous years had shown that this was not a confounding variable $[1,7]$. Sex and surveillance scheme were retained in the model but did not change the VE estimates. When looking at effect modification there was no evidence that VE varied by scheme $(p=0.26)$ or age $(p=0.50)$.

\section{Discussion}

The early experience of the influenza season in the UK [5] and in a number of European Union (EU) Member States, the United States (US) and Canada [12] presents an opportunity for the generation of interim 
TABLE 1

Details for influenza A and B cases and controls originally considered for the mid 2012/13 season trivalent seasonal influenza vaccine effectiveness analysis, United Kingdom, 1 October 2012-4 January $2013(\mathrm{n}=1,865)$

\begin{tabular}{|c|c|c|c|}
\hline Variable & $\begin{array}{l}\text { Controls } \\
n(\%) \\
(N=1,340)\end{array}$ & $\begin{array}{c}\text { Influenza B cases } \\
n(\%) \\
(\mathrm{N}=399)\end{array}$ & $\begin{array}{c}\text { Influenza A cases } \\
n(\%) \\
(N=126)\end{array}$ \\
\hline \multicolumn{4}{|l|}{ Age group (years) } \\
\hline 45 & $159(12)$ & $29(7)$ & $8(6)$ \\
\hline 5-14 & $91(7)$ & $101(25)$ & $9(7)$ \\
\hline $15-44$ & $589(44)$ & $166(42)$ & $80(63)$ \\
\hline $45-64$ & $324(24)$ & $89(22)$ & $25(20)$ \\
\hline$\geq 65$ & $172(13)$ & $14(4)$ & $4(3)$ \\
\hline Missing & $5(0)$ & o (o) & o (o) \\
\hline \multicolumn{4}{|l|}{ Sex } \\
\hline Male & $552(41)$ & $167(42)$ & $55(44)$ \\
\hline Female & $774(58)$ & $223(56)$ & $70(56)$ \\
\hline Missing & $14(1)$ & $9(2)$ & $1(1)$ \\
\hline \multicolumn{4}{|l|}{ Month of sample collection } \\
\hline October & $230(17)$ & $8(2)$ & $13(10)$ \\
\hline November & $553(41)$ & 49 (12) & $23(18)$ \\
\hline December & $543(41)$ & $333(83)$ & $90(71)$ \\
\hline January & $14(1)$ & $9(2)$ & $\mathrm{o}(\mathrm{o})$ \\
\hline \multicolumn{4}{|l|}{ Surveillance scheme } \\
\hline RCGP & $638(48)$ & $271(68)$ & $48(38)$ \\
\hline SMN & $216(16)$ & $48(12)$ & $29(23)$ \\
\hline HPS & $424(32)$ & $40(10)$ & $47(37)$ \\
\hline Wales & $22(2)$ & $18(5)$ & $1(1)$ \\
\hline Northern Ireland & $40(3)$ & $22(6)$ & $1(1)$ \\
\hline \multicolumn{4}{|l|}{ Risk Group } \\
\hline No & $923(69)$ & $315(79)$ & $98(78)$ \\
\hline Yes & $301(22)$ & $56(14)$ & $14(11)$ \\
\hline Missing & $116(9)$ & $28(7)$ & $14(11)$ \\
\hline \multicolumn{4}{|c|}{ Interval between onset and sampling (days) } \\
\hline $0-1$ & $148(11)$ & $31(8)$ & 19 (15) \\
\hline $2-4$ & $495(37)$ & $202(51)$ & $63(50)$ \\
\hline $5-7$ & $325(24)$ & $92(23)$ & $25(20)$ \\
\hline $8-14$ & $197(15)$ & $23(6)$ & $9(7)$ \\
\hline $15-29$ & $59(4)$ & $7(2)$ & $1(1)$ \\
\hline$\geq 29$ & $21(2)$ & $6(2)$ & $1(1)$ \\
\hline Missing onset date & $95(7)$ & $38(10)$ & $8(6)$ \\
\hline \multicolumn{4}{|l|}{ Vaccination status (TIV 2012/13) } \\
\hline Unvaccinated & $996(74)$ & $354(89)$ & $110(87)$ \\
\hline Vaccinated $0-13$ days ago ${ }^{a}$ & $37(3)$ & $1(0)$ & o (o) \\
\hline Vaccinated $\geq 14$ days ago $0^{a}$ & $179(13)$ & $23(6)$ & $9(7)$ \\
\hline Vaccinated timing not known & $47(4)$ & $6(2)$ & $2(2)$ \\
\hline Missing & $81(6)$ & $15(4)$ & $5(4)$ \\
\hline
\end{tabular}

HPS: Health Protection Scotland; RCGP: Royal College of General Practitioners' surveillance scheme; SMN: Health Protection Agency (HPA) Specialist Microbiology Network; TIV: trivalent seasonal influenza vaccine.

${ }^{a}$ This refers to the time interval between vaccination time and time of symptom onset or swab.

assessment of seasonal influenza vaccine effectiveness. This can be used to inform those countries yet to experience a significant season and to add to the evidence base around choice of vaccine composition for the northern hemisphere influenza season of 2013/14. Unlike the pattern in the current season in North America, where influenza activity has been dominated by influenza $A\left(\mathrm{H}_{3} \mathrm{~N}_{2}\right)$ followed by influenza $B$, the early influenza season experienced across the UK has been dominated by influenza B cases with noted homogeneity in this pattern of laboratory detection in each country (with the exception of the Scottish scheme in which influenza $A\left(\mathrm{H}_{3} \mathrm{~N}_{2}\right)$ cases appear to be in slight excess). Influenza B viruses from both the B/Yamagata 
TABLE 2

Selecting participants with known symptom onset of influenza-like illness $(n=1,865)$ for the 2012/13 trivalent seasonal vaccine effectiveness analysis, United Kingdom, 1 October 2012-4 January 2013

\begin{tabular}{|c|c|c|c|c|}
\hline Participants & Controls & Influenza B cases & Influenza A cases & Total \\
\hline With known date of symptom onset ${ }^{a}$ & 1,340 & 399 & 126 & 1,865 \\
\hline With interval from symptom onset to sampling 229 days & -21 & -6 & -1 & -28 \\
\hline Missing vaccination history ${ }^{b}$ & -80 & -15 & -4 & -99 \\
\hline Vaccinated $0-13$ days before symptom onset ${ }^{c}$ & -36 & -1 & -0 & -37 \\
\hline Remaining for vaccine effectiveness analysis & 1,203 & 377 & 121 & 1,701 \\
\hline
\end{tabular}

The "-" sign indicates that the respective participant numbers were excluded from the vaccine effectiveness analysis.

a When the symptom onset date was missing the date when the participant was swabbed was used.

b Numbers exclude the participants with missing vaccination history who additionally had an interval from symptom onset to sampling $>29$ days.

Numbers exclude the participants vaccinated o-13 days before symptom onset who additionally had an interval from symptom onset to sampling $>29$ days and a missing vaccination history.

\section{TABLE 3}

Number of cases versus controls for influenza A and/or B according to 2012/13 trivalent seasonal influenza vaccine vaccination status and vaccine effectiveness (crude and adjusted ${ }^{\mathrm{a}}$ ) estimates, United Kingdom,

1 October 2012-4 January 2013

\begin{tabular}{|c|c|c|c|c|}
\hline Type of influenza & Vaccination status & Cases/controls & Crude VE \% $(95 \% \mathrm{Cl})$ & Adjusted $^{\mathrm{a}}$ VE \% $(95 \% \mathrm{Cl})$ \\
\hline \multirow{2}{*}{$A$ and $B$} & Unvaccinated & $459 / 979$ & \multirow{2}{*}{$63(47$ to 74$)$} & \multirow{2}{*}{51 (27 to 68$)$} \\
\hline & Vaccinated & $39 / 224$ & & \\
\hline \multirow{2}{*}{ A } & Unvaccinated & $110 / 979$ & \multirow{2}{*}{56 (17 to 77$)$} & \multirow{2}{*}{$49(-2$ to 75$)$} \\
\hline & Vaccinated & $11 / 224$ & & \\
\hline \multirow{2}{*}{ B } & Unvaccinated & $349 / 979$ & \multirow{2}{*}{$65(47$ to 77$)$} & \multirow{2}{*}{52 (23 to 70$)$} \\
\hline & Vaccinated & $28 / 224$ & & \\
\hline
\end{tabular}

VE: Vaccine effectiveness.

adjusted for age-group, sex, month and surveillance scheme.

and $B /$ Victoria lineages have co-circulated during the 2012/13 influenza season in the UK, with the majority of influenza $B$ isolates antigenically characterised to date belonging to the B/Yamagata lineage [5].

This is the fifth season in which the UK pooled estimation of TIV VE has been undertaken $[1,7]$. The data quality for the pooled analysis even at this interim analysis stage is deemed high with few missing data field entries.

This observational study of interim influenza VE for TIV against laboratory-confirmed influenza infection in primary care in the UK 2012/13 winter season, which would appear at this juncture to be a medium intensity influenza season with influenza B the dominant circulating strain, has two key findings: reassuringly the northern hemisphere $2012 / 13$ TIV appears to offer moderate protection against the circulating influenza $B$ strain; the point estimate for the TIV VE against influenza $A$ is based on smaller numbers and, though not statistically significant at this stage, suggests a similar moderate level of protection.

These UK interim results which are adjusted for age, sex and calendar month within the season are consistent with the crude TIV VE reported in recent weeks in Morbidity and Mortality Weekly Report (MMWR) from this season's US experience to date [13] in which their season has been dominated by influenza $A\left(\mathrm{H}_{3} \mathrm{~N}_{2}\right)$ and with the overall adjusted VE estimate reported from Canada [14]. Indeed the UK crude VE estimates prior to adjustment for age appear near identical to those in the US.

While the TIV VE for influenza B is statistically significant one should keep in mind that there is a reliance on a trivalent vaccine with only one influenza B component, which in any given influenza season may offer limited protection against another influenza $B$ lineage not targeted by the vaccine [15]. The availability of quadrivalent seasonal influenza vaccines licensed for use in the EU [16] would mean that this can be potentially averted. Work needs to be undertaken to demonstrate whether introduction of these vaccines would be cost-effective.

In conclusion, this study undertaken mid-season provides good evidence that this season's TIV provides protection against laboratory-confirmed influenza B infection and more limited evidence of likely protection against laboratory-confirmed influenza $A$ infection 
in the patients attending their GP with influenza like illness in the UK. It is important to note that more precision in this estimate will be available at the end of the season, together with the ability to obtain agestratified estimates. Vaccination with the seasonal influenza vaccine remains the best protection against influenza. The results are consistent with a protective benefit from seasonal influenza vaccine. Within the UK and beyond, particularly in those countries who are either still early in their influenza season or who have evidence of continuing influenza transmission, it is important to stress that it is not too late to be vaccinated this season and individuals in clinical groups eligible for vaccination who have yet to be vaccinated should be encouraged to get vaccinated.

\section{Acknowledgements}

We are grateful to the many primary care physicians in England, Wales, Northern Ireland and Scotland who supplied the clinical information on their patients; to the staff of the HPA Respiratory Virus Unit, the HPA regional microbiology laboratories, Public Health Wales Specialist Virology Centre, the West of Scotland Specialist Virology Centre and the Regional Virus Laboratory, Belfast who undertook analysis of specimens. We thank the staff of the Health Protection Agency, RCGP, Public Health Wales, Public Health Agency Northern Ireland and Health Protection Scotland teams who coordinate the GP schemes, in particular Joy Field from the HPA; Richard Lewis and Hannah Evans from PHW; Catherine Frew, Rory Gunson \& Celia Aitken from WoSSVC and Arlene Reynolds, Louise Primrose-Shaw and Lesley McGuire from HPS for overseeing data collection.

\section{Funding}

The infrastructure and methodology to enable the work was developed as part of a European Centre for Disease Control and Prevention (ECDC) funded project "I-MOVE". The I-MOVE group continues to be co-ordinated by Epi-Concept (http:// www.epiconcept.fr/).

\section{Conflicts of interest}

All authors have completed the Unified Competing Interest form at http://www.icmje.org/coi_disclosure.pdf (available on request from the corresponding author) and declare that DM Fleming has received funding to attend influenza related meetings and has received consultancy fees from influenza vaccine manufacturers who might have an interest in the submitted work in the previous 3 years. In addition, the Virus Reference Department of the Health Protection Agency receives funding from a variety of vaccine manufacturers who might have an interest in the submitted work. All other authors declare they have no conflicts of interest.

\section{Authors' contributions}

Jim McMenamin led the writing of the rapid communication. All authors provided contribution to the rapid communication and approved the final version. Nick Andrews and Chris Robertson undertook the statistical analysis on which the rapid communication is based. Richard Pebody, Nick Andrews, Douglas Fleming, John Watson, Jim McMenamin and Chris Robertson were involved in the original methodological design but all other authors have had a role in modification of this design over the years.

\section{References}

1. Pebody RG, Andrews N, McMenamin J, Durnall H, Ellis J, Thompson $\mathrm{Cl}$, et al. Vaccine effectiveness of $2011 / 12$ trivalent seasonal influenza vaccine in preventing laboratoryconfirmed influenza in primary care in the United Kingdom: evidence of waning intra-seasonal protection . Euro Surveill. 2013;18(5):pii=20389. Available from: http://www. eurosurveillance.org/ViewArticle.aspx?Articleld=20389

2. Health Protection Agency (HPA). Surveillance of influenza and other respiratory viruses in the UK: 2011-2012 report. London: HPA; Jun 2012. Available from: http://www.hpa.org.uk/webw/ HPAweb\&HPAwebStandard/HPAweb_C/1317134576275

3. Jiménez-Jorge S, de Mateo S, Pozo F, Casas I, García Cenoz M, Castilla J et al. Early estimates of the effectiveness of the $2011 / 12$ influenza vaccine in the population targeted for vaccination in Spain, 25 December 2011 to 19 February 2012 Euro Surveill. 2012;17(12):pii=20129. Available from: http:// www.eurosurveillance.org/ViewArticle.aspx?Articleld $=20129$

4. World Health Organization (WHO). Recommended composition of influenza virus vaccines for use in the 2012-2013 northern hemisphere influenza season. Geneva: WHO; 23 Feb 2012. Available from: http://www.who.int/influenza/vaccines/virus/ recommendations/2012_13_north/en/index.html

5. Health Protection Agency (HPA). Weekly National Influenza Report. 17 January 2013 - Week 3 report (up to week 2 data). London: HPA; 17 Jan 2013. Available from: http://www.hpa.org. uk/webc/HPAwebFile/HPAweb C/1317137745086

6. The Chief Medical Officer, the director of nursing and the chief pharmaceutical officer for England. The flu immunisation programme 2012/13. London: Department of Health; 3 May 2012. Available from: http://www.dh.gov. uk/en/Publicationsandstatistics/Lettersandcirculars/ Professionalletters/DH_133888

7. Pebody RG, Andrews N, Fleming DM, McMenamin J, Cottrell S Smyth B, et al. Age-specific vaccine effectiveness of seasona $2010 / 2011$ and pandemic influenza $A\left(\mathrm{H}_{1} \mathrm{~N}_{1}\right) 2009$ vaccines in preventing influenza in the United Kingdom. Epidemiol. Infect. 2012:1-11.

8. Gunson R, Maclean A, Davies E, Bennett S, Miller R, Carman WF. Development of a multiplex real-time RT-PCR that allows universal detection of influenza $A$ viruses and simultaneous typing of influenza A/H1N1/2009 virus. J Virol Methods. 2010;163(2):258-61.

9. Ellis J, Iturriza M, Allen R, Bermingham A, Brown K, Gray J, Brown D. Evaluation of four real-time PCR assays for detection of influenza $A\left(\mathrm{H}_{1} \mathrm{~N}_{1}\right)$ v viruses. Euro Surveill. 2009;14(22):pii=19230. Available from: http://www. eurosurveillance.org/ViewArticle.aspx?Articleld=19230

10. To KK, Chan KH, Li IW, Tsang TY, Tse H, Chan JF, et al. Viral load in patients infected with pandemic $\mathrm{H}_{1} \mathrm{~N}_{1} 2009$ influenza $\mathrm{A}$ virus. J Med Virol. 2010;82(1):1-7.

11. Stephenson I, Democratis J, Lackenby A, McNally T, Smith J, Pareek $M$, et al. Neuraminidase inhibitor resistance after oseltamivir treatment of acute influenza $A$ and $B$ in children. Clin Infect Dis. 2009;48(4):389-96.

12. European Centre for Disease Prevention and Control (ECDC). Weekly influenza surveillance overview, Stockholm: ECDC; 18 Jan 2013. Available from: http://ecdc.europa.eu/en/ publications/Publications/130118_SUR_Weekly_Influenza_ Surveillance_Overview.pdf

13. Centers for Disease Control and Prevention (CDC). Early estimates of seasonal influenza vaccine effectiveness United States, January 2013. MMWR Morb Mortal Wkly Rep. 2013;62:32-5. http://www.cdc.gov/mmwr/preview/mmwrhtml/ mm6202a4.htm?s_cid=mm6202a4_w

14. Skowronski DM, Janjua NZ, De Serres G, Dickinson JA, Winter A, Mahmud SM, et al. Interim estimates of influenza vaccine effectiveness in 2012/13 from Canada's sentinel surveillance network, January 2013. Euro Surveill. 2013;18(5): pii=20394. Available from: http://www.eurosurveillance.org/ViewArticle. aspx?Articleld $=20394$

15. Ambrose CS, Levin MJ. The rationale for quadrivalent influenza vaccines. Hum Vaccin Immunother. 2012;8(1):81-8.

16. European Medicines Agency (EMA). Amended EU recommendations for the seasonal influenza vaccine composition for the season 2012/2013. London: EMA; $19 \mathrm{Apr}$ 2012. Available from: - http://www.ema.europa.eu/docs/en GB/document_library/Other/2012/03/WC500124221.pdf 